\title{
Medida de similaridad entre imágenes de marcas de ganado mediante distribuciones de forma
}

\author{
Germán Sánchez Torres* \\ Manuel E. Rodríguez García*
}

Recibido: 09/04/2013 - Aceptado: 27/06/2014

\begin{abstract}
Resumen
Este documento reporta los resultados de una investigación orientada hacia el diseño de un método de tratamiento de imágenes digitales para la automatización de los procesos de registro y control de marcas de ganado requeridas por las regulaciones del sector ganadero en Colombia. El método permite automatizar los procesos de búsqueda y de comparación necesarios para garantizar la unicidad de las marcas dentro de un sistema asistido por computadora. Se inicia con la generación de un histograma estimado de la geometría de la marca, lo que permite comparar y detectar similitudes entre las figuras previamente almacenadas, mediante una métrica de similitud basada en la distancia de Minkowski. Los resultados obtenidos indican que el método es adecuado para realizar un proceso de discriminación de dichas imágenes, reducir las ambigüedades y garantizar la unicidad de los registros. Los resultados obtenidos y un análisis de su aplicación son reportados.
\end{abstract}

Palabras clave: automatización, distribuciones de forma, hierro quemador, similitud de imágenes 2D.

\footnotetext{
Autor de correspondencia, I. S., M.Sc., Doctor en Ingeniería. Profesor Asistente, Facultad de Ingeniería, Universidad del Magdalena. Grupo de Investigación y Desarrollo en Nuevas Tecnologías de la Información y la Comunicación. Correo electrónico: gsanchez@unimagdalena.edu.co. Teléfono: (57) 5- 4301292 Ext. 1138, Carrera 32 N. ${ }^{\circ} 22-08$, Ed. Docente, Cub. 3D401, Santa Marta - Magdalena, Colombia

** Ingeniero de Sistemas, Facultad de Ingeniería, Universidad del Magdalena. Grupo de Investigación y Desarrollo en Nuevas Tecnologías de la Información y la Comunicación. Correo electrónico: novdarwin@hotmail.com. Carrera 32 N. ${ }^{\circ} 22-08$, Santa Marta, Colombia
} 


\section{Cattle marks similarity measure by shape distributions}

\section{Abstract}

This paper reports the investigation results addressed to design of a digital image processing method for automate the registration and control process of livestock marks according to Colombian regulations livestock sector. It allows automate the process of search and comparison required for livestock mark uniqueness assurance on a computer assisted system. First, a histogram is generated based on statistical distribution of geometrical information, which permit to detect similarities between registered marks by means Minkowski-based metric. The results show that the method allows discriminating between images using a digital signature from a Shape Distribution the geometric structure reducing ambiguity and ensuring the uniqueness of registered marks. We show the results and make an analysis of system application.

Key words: Process automation, shape distribution, Hot-Iron, 2d image similarity. 


\section{INTRODUCCIÓN}

La identificación animal es importante para la correcta aplicación de medidas orientadas a la prevención y el control de crisis agroalimentarias que podrían estar originadas en las condiciones de salud en los animales destinados para el consumo. La identificación individualizada posibilita la trazabilidad al aplicar un registro completo del desarrollo del animal. La trazabilidad, definida como la capacidad de determinar el origen alimenticio y la identificación del mismo a lo largo de toda o parte de su vida útil [1, 2], constituye un requisito exigido por la comunidad internacional en escenarios de exportación como el mercado bovino colombiano.

Las tendencias encontradas para la realización de la trazabilidad animal son diversas, y varían desde la clásica y tradicional marca con fuego, hasta las modernas implantaciones de dispositivos electrónicos [3, 4] o las huellas de ADN [5]. Existen restricciones para la aplicación de algunas de estas técnicas fundamentadas en diversos motivos; los dispositivos electrónicos presentan el riesgo de afectar al consumidor al entrar en la cadena alimenticia, mientras que las marcas con fuego son rechazadas por estrictas medidas de protección del bienestar animal. Pese a esto, el panorama nacional es tradicional, debido a que las marcas con fuego constituyen la principal estrategia en las regiones colombianas para identificar un animal. En Colombia se evidencia aún un precario desarrollo de las herramientas tecnológicas puestas a disposición para tal fin.

Una vez se ha seleccionado una marca (ver figura 1), esta debe ser registrada ante las entidades territoriales mediante un procedimiento denominado Registro de Hierro Quemador. En Colombia, el proceso de Registro de Hierro Quemador, "Es la inscripción de la marca con que la persona, ya sea natural ojurídica, demuestra que es propietaria de ganados u otros, acostumbrados a marcarlos" [6]. El proceso de registro de hierro quemador es un trámite que se hace de forma manual en todos los municipios de la nación colombiana y consiste en llevar un elemento metálico que representa la marca ganadera a la oficina encargada del trámite en la entidad territorial [7]. Esta marca es registrada mediante una copia manual de la imagen generada con dicho elemento.

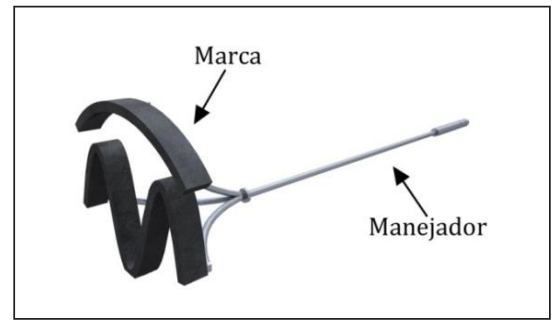

Figura 1. Elemento de hierro destinado a la marca de ganado Fuente: elaboración propia 
El proceso de registro inicia con la comprobación de que no existe la nueva marca en los registros vigentes. Esta decisión es tomada mediante una búsqueda realizada sobre un conjunto de libros físicos que contienen las figuras inscritas con anterioridad (ver figura 2a).

Este proceso de búsqueda se efectúa en forma manual, por lo que depende de la capacidad visual de quien lleva a cabo la operación para garantizar su efectividad, del estado en que se encuentren los libros de registro, y de la calidad y claridad con la que se tomaron dichos registros. Si no es encontrada una coincidencia con alguna marca registrada previamente, se procede a diligenciar un Acta de registro de Hierro Quemador (ver figura 2b), que establece que esta marca ganadera pertenecerá al tramitador y que tendrá vigencia en ese municipio.
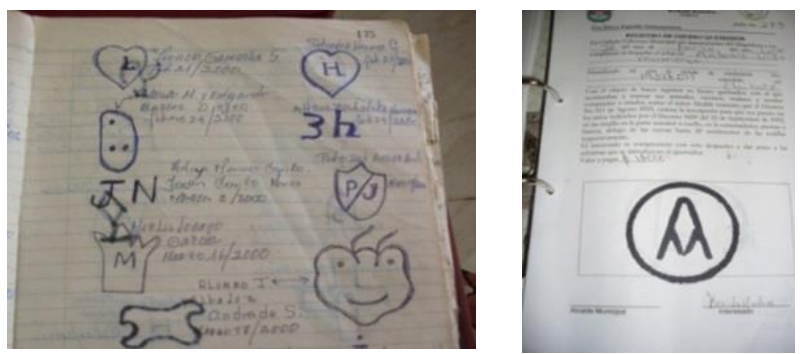

Figura 2: (a) Libro de registros, (b) Acta de registro.

Fuente: elaboración propia

El proceso de registro, al no poseer sistematización, ocasiona la búsqueda sobre datos dispersos y voluminosos, con procedimientos no estructurados y poco efectivos, dando lugar a deficiencias en la efectividad del proceso. Adicionalmente, este procedimiento es local, lo que impide la aplicación de estrategias de seguimiento y control del ganado a niveles departamentales o nacionales.

Este trabajo está dirigido hacia la proposición de un método para la sistematización del proceso de registro del hierro quemador. El interés principal se centra en el proceso de digitalización y búsqueda de una nueva marca dentro del conjunto de marcas registradas. Este proceso exige una comparación directa con las marcas previamente registradas para garantizar la unicidad del registro. La unicidad se garantiza mediante una métrica establecida de similitud entre imágenes. Dicha métrica es estimada sobre una firma digital de cada figura, que constituye una cadena de enteros que representa la forma de la figura, extraída de la geometría intrínseca de la imagen. Para esto, cada nueva imagen es almacenada de manera digital junto con su respectiva firma estimada. La firma es una representación de la distribución de las distancias de un conjunto de puntos que describen la geometría del esqueleto de la figura. Cada intento de registro 
sugiere un proceso automático de comparación de la nueva imagen con un conjunto de imágenes registradas y previamente almacenadas. La métrica utilizada para determinar la similitud de firmas es la distancia de Minkowski [8]. Los resultados muestran que el método es adecuado para reducir el conjunto de posibles marcas registradas coincidentes con un nuevo registro.

\section{MATERIALES Y MÉTODOS}

El método propuesto consta de varias etapas, algunas de las cuales corresponden a etapas clásicas del procesamiento de imágenes digitales. El procedimiento inicia con la adquisición de la imagen, a la cual se le aplica procedimiento de preprocesamiento que genera una representación digital sobre la cual se estima el esqueleto de la forma de la figura que es empleado en la generación de la firma o secuencia representativa de la imagen. Una vez adquirida esta firma es utilizada para los procedimiento de comparación que generan un conjunto de posibles coincidencias (ver figura 3). La mayor relevancia está centrada en el procedimiento para la generación de una firma de la forma de la marca obtenida desde un modelo 2D como una distribución de probabilidad de una función de forma que mide las propiedades geométricas del símbolo que representa la marca.

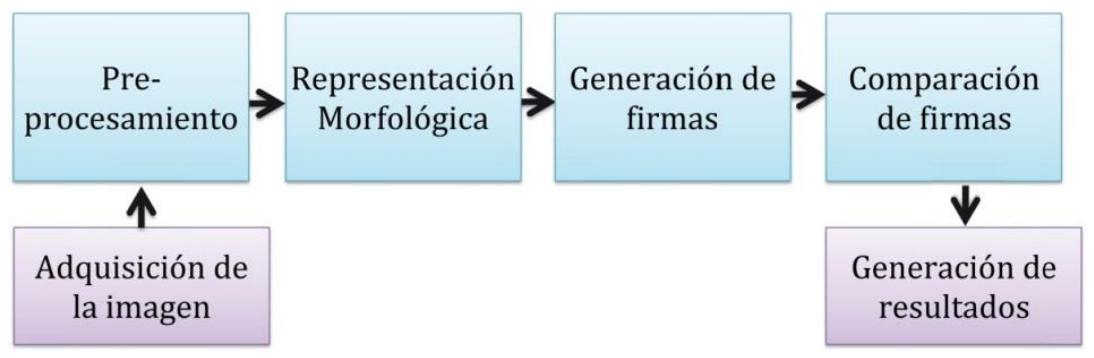

Figura 3: Algoritmo del Método.

Fuente: elaboración propia

Adquisición de la imagen: Dado que actualmente no existe digitalización alguna en este proceso, se propone utilizar imágenes de fotografía digital en formato $P N G$ (portable network graphics) [9], que no superen los 800x800 píxeles.

Pre-procesamiento: Generalmente, el preprocesamiento incluye operaciones de bajo nivel de abstracción, cuyo objetivo es mejorar los datos en la imagen, reducir las distorsiones o resaltar características de la imagen, útiles en alguna transformación posterior. En este contexto, se hace una transformación a escala de grises, principalmente porque no existe información de color relevante en la imagen adquirida, y se binariza la imagen, separando la zona de interés del fondo. 
Representación morfológica: Una vez se tiene la región de interés se aplica un descriptor morfológico para representar las marcas con el menor número de píxeles posibles conservando sus características esenciales [10]. Pese a los diferentes operadores, se seleccionó el operador Skeleton o Esqueleto, debido a que la estructura morfológica del operador esqueleto suministra información directamente relacionada con la forma, el tamaño y la orientación (ver figura 4) de la figura. De tal forma que, dada una imagen $I$, la transformación morfológica es definida como [11]:

$$
S k(I)=\bigcup_{n=0}^{N} S k_{n}(I)
$$

Cada uno de los $S k_{n}(I)(n=0,1, \cdots, N)$ pueden ser estimados desde I, a partir de un elemento de estructuras B, así:

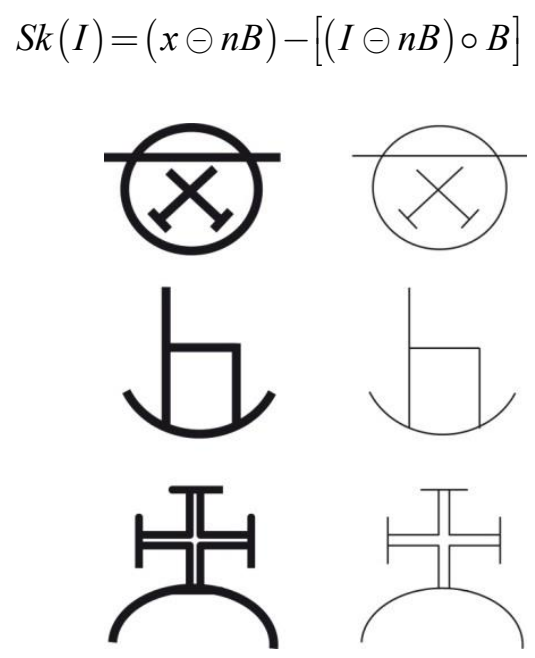

Figura 4: Efecto de la aplicación del descriptor morfológico.

Fuente: elaboración propia

Generalmente, $N$ es el tamaño máximo del elemento de estructuras después del cual una siguiente aplicación del funcional de erosión, convierte a $I$ en un conjunto vacío. Diferentes algoritmos para la implementación computacional de este operador han sido propuestos. En [12] se presenta un algoritmo eficiente para estimar el esqueleto de una imagen.

Obtención de la firma: La generación de firmas de imágenes intenta determinar una medida o conjunto de estas que representen con unicidad cada imagen. Estas han sido utilizadas en dirección a solucionar diferentes problemas como la búsqueda por similaridad [12], autentificación y detección de duplicaciones [13]. 
La representación morfológica brinda la geometría de una región relacionando el tamaño, la posición, la orientación y la forma $[14,15]$. Como en este caso la región de interés es el conjunto de píxeles comprendidos en el interior del esqueleto de la marca, se utilizó la técnica de la construcción de distribuciones de forma, la cual representa las características geométricas del contenido de la imagen mediante una función de forma $[8,16]$. La base sobre la cual el método de generación de formas se sustenta es la selección del funcional de forma, es decir, una función que genere una distribución que garantice la unicidad de la firma. Dentro de las funciones de forma basadas en geometría se encuentran (ver figura 5):

- A3: Basada en él ángulo entre tres puntos aleatorios sobre la imagen.

- D1: Basada en la distancia entre un punto fijo y un punto aleatorio en la superficie.

- D2: Basada en la distancia de dos puntos aleatorios.

- D3: Basada en la raíz cuadrada del área formada por tres puntos aleatorios.

- D4: Basada en el cubo cuadrado del volumen del tetraedro entre cuatro puntos aleatorios.

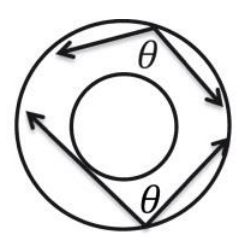

A3

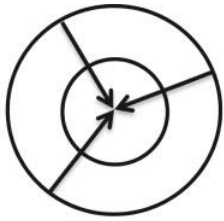

D1

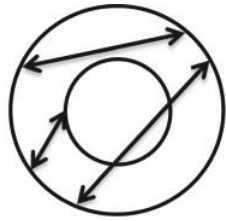

D2

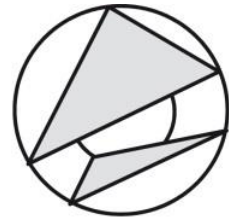

D3

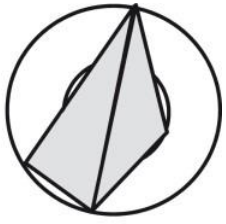

D4

Figura 5. Representación gráfica de las funciones de forma típicas

Fuente: [8]

El procedimiento específico consiste en tomar parejas de puntos al azar en la imagen y calcular su distancia euclidiana. Este proceso garantiza que la función de forma tenga invariancia bajo movimientos rígidos y espejos de la proyección de imagen. El muestro aleatorio asegura que las distribuciones de forma no son sensibles a pequeñas perturbaciones. En este caso, en virtud de la invariancia de escala, puede ser añadida por la estandarización de las distribuciones de forma antes de hacer comparaciones.

\section{CONSTRUCCIÓN DE LA DISTRIBUCIÓN DE FORMA}

Una vez seleccionada la función de forma, D2 para nuestro caso, se calcula y almacena una representación de su distribución. Primero se evalúan $N$ muestras, o parejas de 
puntos al azar, sobre las cuales se calculan las distancias; este muestreo es utilizado para construir un histograma de $B$ categorías de agrupación. Con el histograma, se construye una función lineal $\vartheta=\left\{\varnothing_{0}, \cdots, \varnothing_{v}\right\}$ a trozos con $V$ vértices equidistantes $V \leq B$ (ver figura 6), que constituye la firma, y está formada de una secuencia de números enteros correspondientes al valor del histograma en cada vértice.

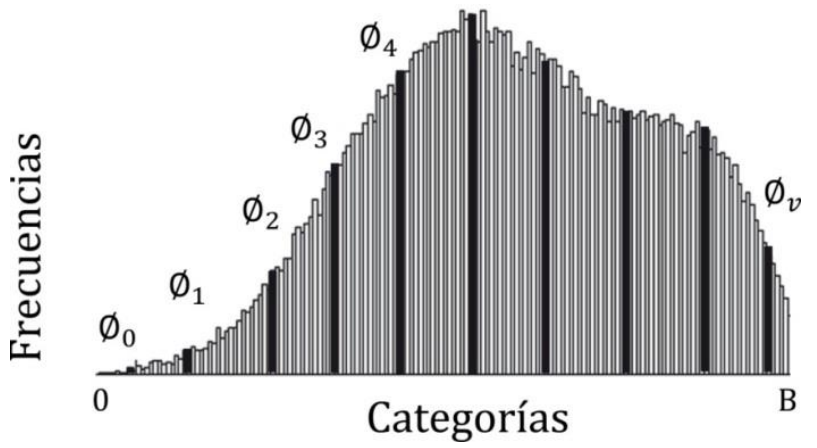

Figura 6. Histograma y construcción de la función discreta por pedazos.

Fuente: elaboración propia

\section{COMPARACIÓN ENTRE DISTRIBUCIÓN DE FORMA}

Cada imagen de marca debe ser almacenada con su firma. Cada intento de inserción de una nueva imagen requiere la comparación con las imágenes existentes para determinar su unicidad. Esta comparación es realizada mediante la similitud de las secuencias enteras tomadas como firmas. Existen diferentes métricas para estimar la disimilitud de funciones; se ha seleccionado un procedimiento con enfoque estadístico para la disimilitud entre dos firmas, basado en una métrica denominada distancia de Minkowski definida como:

$$
\operatorname{Dis}(f 1, f 2)=\left\{d_{p}(f 1, f 2)\right\}
$$

Donde todo $(f 1, f 2)$, constituye un par de firmas, $p$ puede ser un número entero $p=1,2, \cdots \infty$. La función $d_{p}(f 1, f 2)$ es definida así:

$$
d_{p}(f 1, f 2)=\left(\sum_{i=1}^{n=V}\left|f 1_{i}-f 2_{i}\right|^{p}\right)^{1 / p}
$$

Donde los $f 1_{i}$ y los $f 2_{i}$ representan los vértices de cada firma. Esta distancia cuantifica la disimilitud entre dos marcas. Dado que los histogramas pueden estar en 
escalas diferentes es necesario aplicar algún método de normalización para garantizar la consistencia en la comparación. Finalmente, las imágenes similares se encuentran mediante un procedimiento de optimización que tenga en cuenta las diferencias entre escalas de las distribuciones, así:

$$
\min _{V} d(H f 1, H f 2)
$$

Donde, $\mathrm{H} f 1, \mathrm{H} f 2$, son histogramas normalizados de $f 1$ y $f 2$. $\mathrm{H} f 1$ es fija, o es la nueva marca que se desea registrar, y $\mathrm{H} f 2$ constituye las imágenes similares en un conjunto de búsqueda. El procedimiento completo es presentado en el algoritmo 1.

Algoritmo 1. Función de disimilitud

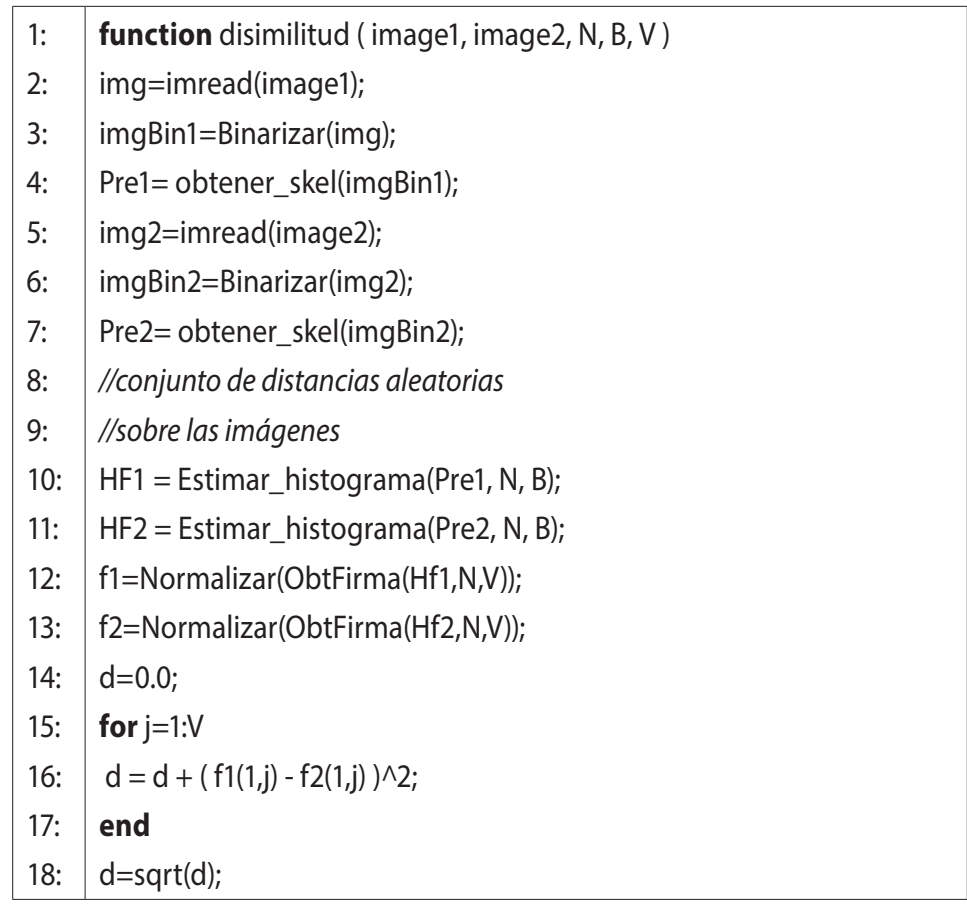

\section{RESULTADOS Y DISCUSIÓN}

Inicialmente, se estudió la influencia del tamaño de la muestra para generar una distribución de forma, dado el componente aleatorio al seleccionar los puntos dentro de cada imagen. Por lo tanto, para un conjunto de 20 imágenes (ver figura 7), se calculó 200 veces la disimilitud entre imágenes iguales variando solo el tamaño de $N$. El comportamiento indica que la disimilitud es inversamente proporcional al tamaño de 
la muestras (ver figura 8). Sin embargo, el aumento del tamaño de $N$ implica mayor tiempo en la construcción de la distribución. Experimentalmente, se ha encontrado que el uso de $\mathrm{N}=1.024, \mathrm{~B}=64$ categorías, $\mathrm{y} \mathrm{V}=44$ vértices da un buen rendimiento para construir la distribución de forma con una varianza lo suficientemente baja.
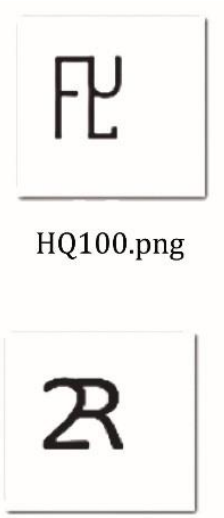

HQ105.png

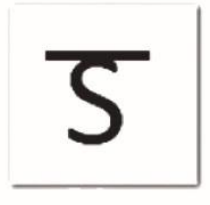

HQ101.png

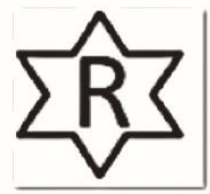

HQ106.png

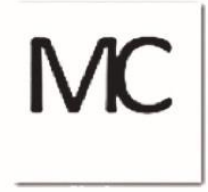

HQ102.png

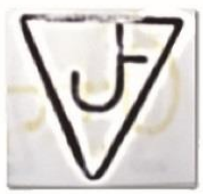

HQ107.png

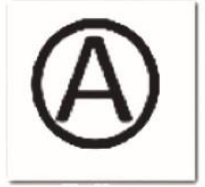

HQ103.png

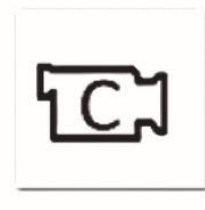

HQ108.png

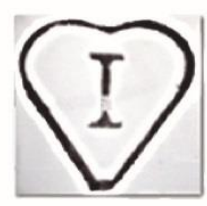

HQ104.png

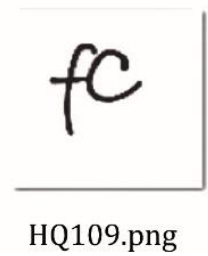

Figura 7. Ejemplos de marcas utilizadas

Fuente: elaboración propia

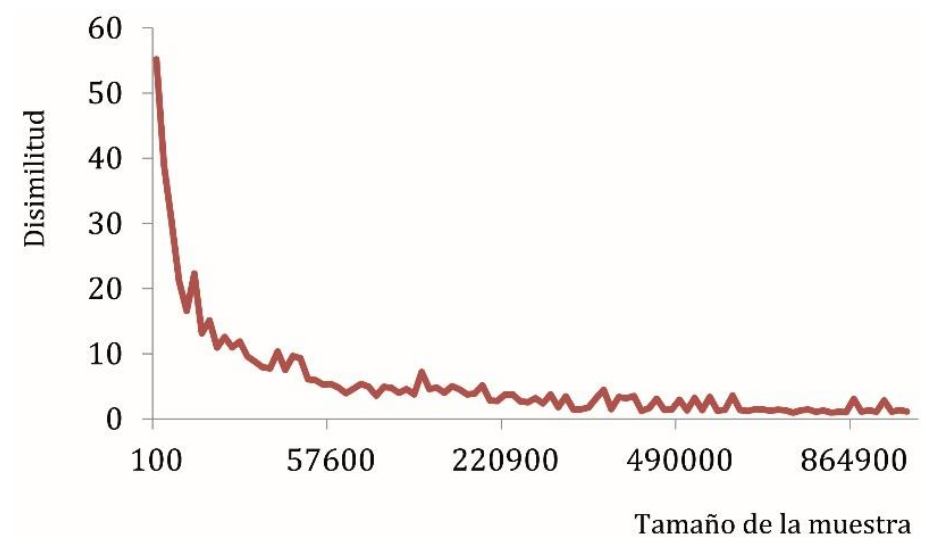

Figura 8: Comportamiento de la estimación de la medida de disimilitud en relación al tamaño del muestreo

Fuente: elaboración propia

Con el valor de estos parámetros, se calculó la disimilitud de pares de imágenes iguales para estimar la sensibilidad del factor aleatorio, y se encontró que el promedio de las varianzas fue de 0,18033 . Finalmente, se aplicó la métrica de disimilitud entre 
imágenes diferentes para estimar la efectividad del método. El promedio de disimilitud entre pares de imágenes diferentes fue de 37,3.

La figura 9 muestra el resultado de la medida de similitud entre pares de marcas homogéneos, como transformaciones espejos y translaciones, y pares no homogéneos conformados por la selección de marcas diferentes. El comportamiento apreciable en la estimación de la disimilitud, hace que puedan diferenciarse marcas cuya forma, representada por la representación en esqueleto de su geometría, sea diferente.

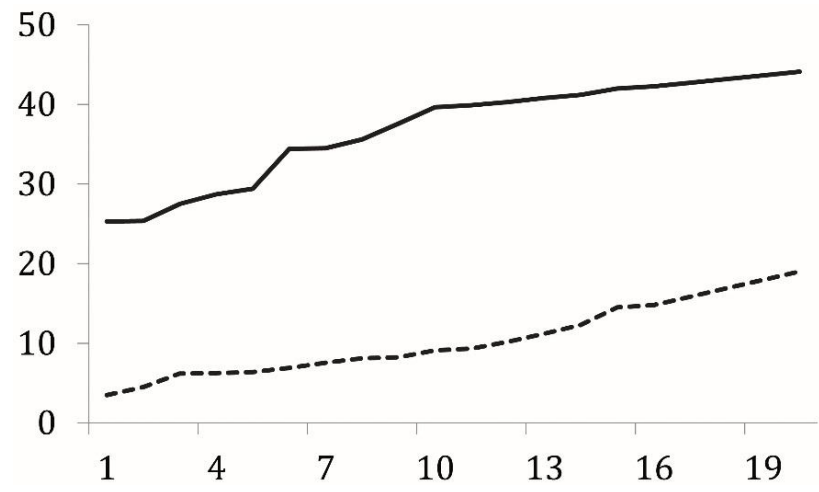

Figura 9. Comparación de disimilitud entre pares de marcas homogéneas (línea punteada) y diferentes (líneas continua)

Fuente: elaboración propia

\section{CONCLUSIONES}

Se propuso el uso de un operador de forma basado en la distribución de las distancias entre puntos de la imagen como elemento discriminante entre figuras que representan marcas de ganado para hacer una discriminación suficiente entre marcas disimiles.

La precisión en los resultados está relacionada con el número de vértices $V$ en el vector de enteros de la firma. El tiempo computacional para construir la distribución de la muestra es directamente proporcional al número de distancias calculadas, por lo que $N$ debe elegirse con precaución teniendo en cuenta una relación entre precisión y costo computacional.

Finalmente, este método posibilita la discriminación de imágenes de marca de ganado y puede utilizarse como un paso intermedio en una solución computacional general, que esté orientada hacia la automatización de los procedimientos de registro y seguimiento de las marcas de ganado. Sin embargo, es posible que las estructuras geométricas de varias marcas generen medidas que se encuentren cercanas en rela- 
ción con el espacio matemático de la métrica. Esto puede ocurrir si la base de datos de imágenes registradas aumenta su tamaño, debido a que la información contenida en las imágenes de marca de ganado es limitada, y está solo referida a la geometría de la forma que la constituye. En este escenario el método funciona como un reductor del conjunto de imágenes similares, dejando al usuario del sistema la decisión final relacionada con la unicidad de una nueva marca a registrar.

\section{REFERENCIAS}

[1] R. Pedretti, "Trazabilidad de carne bovina en Paraguay", Proyecto de cooperación técnica FAO/TCP/2910 apoyo a la integración agropecuaria en el Mercosur ampliado. Julio, 2004.

[2] R. Fernández, "Trazabilidad alimentaria. Una herramienta decisiva para la seguridad y la protección de los consumidores”, Distribución y Consumo. N. ${ }^{\circ}$ 62, pp. 5-10, 2002.

[3] Caja, G., Barillet, F., Nehring, R., Marie, C., Ribó, O., Ricard, E., Lagriffoul, G., Conill, C., Aurel, M.R. y Jacquin, M., "Comparison of different devices for electronic identificatioinn d airy sheep", En: Performance recording of animals. Proceedings of 30» biennial session of the International Committee for Animal Recording, EAAP Publication, n. ${ }^{\circ}$ 87, Wageningen Pers, Wageningen, pp. 349-353, 1996.

[4] Ribó O, Korn C, Meloni U, Cropper M, De Winne P, Cuypers M., "IDEA: a large-scale project on electronic identification of livestock", Rev. Sci Tech Off int Epiz, vol. 20, pp. 426-436. 2001.

[5] R. Felmer, R. Chávez, A. Catrileo, y C. Rojas, "Tecnologías actuales y emergentes para la identificación animal y su aplicación en la trazabilidad animal”, Arch. Med. Vet. Valdivia, Vol. 38, n. ${ }^{\circ}$ 3, 2006.

[6] Portal del Gobierno En Línea. (2010). Trámites y Servicios, Registro de Hierro Quemador. [En línea], acceso 21 noviembre 2012 http://www.gobiernoenlinea.gov.co/web/guest/home/-/ government-services/8233/maximized.

[7] Federación Colombiana de Ganaderos -FEDEGAN-FNG, "Plan Estratégico de la Ganadería Colombiana 2019”, Colombia: Sanmartín Obregón \& Cía., noviembre de 2006.

[8] R. Osada, et al., "Shape Distributions", Princeton University-ACM Transactions on Graphics, vol. 21, N. ${ }^{\circ}$, pp. 807-832, 2002.

[9] G. Roelofs, "PNG Lossless Image Compression" en Lossless Compression Handbook, ed. Khalid Sayood. pp. 371-390. Academic Press, San Diego, 2003.

[10] K. Saeed, M, et al., "K3M: A universal algorithm for image skeletonization and a review of thinning techniques". Int. J. Appl. Math. Comput. Sci. vol. 20, ․ㅜ 2, pp. 317-335. 2010.

[11] R. Osada, T. Funkhouser, B. Chazelle, y D. Dobkin, "An improved image coding algorithm using morphological operator theory", International Conference on Acoustics, Speech, and Signal Processing, ICASSP-91, vol. 4, pp. 2761-2764. 1991. 
[12] P. Morrison y J. Zou, «An effective skeletonization method based on adaptive selection of contour points», Information Technology and Applications, 2005. ICITA 2005. Third International Conference on COMPLETE, vol.1, n. ${ }^{\circ}$, pp.644,649 vol.1, 4-7 July 2005

[13] A. Zhiyong, Z. Zhiyong y Z. Lihua, "Content-Based Image Retrieval Based on the Wavelet Transform and Radon Transform", Industrial Electronics and Applications. On ICIEA 2007. 2nd IEEE Conference, vol. 2, pp.1878-1881. 2007.

[14] C. Wong, M. Bern, y D. Goldberg, "An image signature for any kind of image”, International Conference on Image Processing, vol.1, pp. 409-412. 2002.

[15] E. Gutiérrez, L. Sánchez, R. Alaiz y J. Domínguez., “Utilización de momentos estadísticos y redes neuronales en la clasificación de cabezas de espermatozoides de verraco", En XXV jornadas de Automática, Ciudad Real, pp. 1-6, 2004.

[16] M. Domingo, "Extracción de características”, Universidad Católica de Chile. Departamento de Ciencia de la Computación, Santiago de Chile, Guía académica, 2006.

[17] Y. Liu, H. Zha y H. Qin, “The Generalized Shape Distributions for Shape Matching and Analysis”. IEEE International Conference on Shape Modeling and Applications. 2006. 